\title{
Molecular characterization of the SPL gene family in Populus trichocarpa
}

\author{
Caili Li and Shanfa Lu*
}

\begin{abstract}
Background: SPLs, a family of transcription factors specific to plants, play vital roles in plant growth and development through regulation of various physiological and biochemical processes. Although Populus trichocarpa is a model forest tree, the PtSPL gene family has not been systematically studied.

Results: Here we report the identification of 28 full-length PtSPLS, which distribute on 14 P. trichocarpa chromosomes. Based on the phylogenetic relationships of SPLs in P. trichocarpa and Arabidopsis, plant SPLs can be classified into 6 groups. Each group contains at least a PtSPL and an AtSPL. The N-terminal zinc finger 1 (Zn1) of SBP domain in group 6 SPLs has four cysteine residues (CCCC-type), while Zn1 of SPLs in the other groups mainly contains three cysteine and one histidine residues (C2HC-type). Comparative analyses of gene structures, conserved motifs and expression patterns of PtSPLs and AtSPLs revealed the conservation of plant SPLs within a group, whereas among groups, the P. trichocarpa and Arabidopsis SPLs were significantly different. Various conserved motifs were identified in PtSPLs but not found in AtSPLs, suggesting the diversity of plant SPLs. A total of 11 pairs of intrachromosome-duplicated PtSPLs were identified, suggesting the importance of gene duplication in SPL gene expansion in P. trichocarpa. In addition, 18 of the 28 PtSPLs, belonging to G1, G2 and G5, were found to be targets of miR156. Consistently, all of the AtSPLs in these groups are regulated by miR156. It suggests the conservation of miR156-mediated posttranscriptional regulation in plants.

Conclusions: A total of 28 full-length SPLs were identified from the whole genome sequence of $P$. trichocarpa. Through comprehensive analyses of gene structures, phylogenetic relationships, chromosomal locations, conserved motifs, expression patterns and miR156-mediated posttranscriptional regulation, the PtSPL gene family was characterized. Our results provide useful information for evolution and biological function of plant SPLS.
\end{abstract}

\section{Background}

SPL proteins constitute a diverse family of transcription factors playing vital roles in plant growth and development. SPLs are specific to plants and have a highly conserved SBP (SQUAMOSA PROMOTER BINDING PROTEIN) domain with approximately 78 amino acid residues. The domain contains three functionally important motifs, including zinc finger 1 (Zn1), zinc finger 2 (Zn2), and nuclear location signal (NLS) [1,2]. Genes encoding SPLs were first identified for SBP1 and SBP2 in Antirrhinum majus [3]. Lately, it has been found in various green plants, including single-celled green algae, mosses, gymnosperms, and angiosperms. The results showed that SPLs existed as a large gene family in plants.

\footnotetext{
* Correspondence: sflu@implad.ac.cn

Institute of Medicinal Plant Development, Chinese Academy of Medical Sciences \& Peking Union Medical College, No.151, Malianwa North Road, Haidian District, Beijing 100193, China
}

For instance, the SPL gene family in Arabidopsis, rice, Physcomitrella patens, maize and tomato includes 16, 19, 13, 31 and 15 members, respectively [4-9].

The 16 Arabidopsis SPLs are termed as AtSPL1 to AtSPL16 [2], respectively, of which AtSPL1, AtSPL7, AtSPL12, AtSPL14 and AtSPL16 are relatively large and expressed constitutively, while the others are relatively small and highly expressed in flowers $[4,10]$. Ten of the 16 AtSPLs, including AtSPL2-AtSPL6, AtSPL9-AtSPL11, AtSPL13 and AtSPL15, are regulated by miRNAs belonging to the MIR156 family [11-17]. AtSPL3, AtSPL4 and AtSPL5 contain complementary sequences of miR156 in 3' UTR, and all of them promote vegetative phase change and flowering $[10,14,18]$. AtSPL2, AtSPL10 and AtSPL11 regulate morphological traits of cauline leaves and flowers [19]. Overexpression of miR156b reduces the accumulation of AtSPL2, AtSPL10 and AtSPL11 mRNA [12,14,20]. AtSPL9 and AtSPL15 act redundantly 
in controlling the juvenile-to-adult growth phase transition and leaf initiation rate in Arabidopsis [21]. Six AtSPLs, including AtSPL1, AtSPL7, AtSPL8, AtSPL12, AtSPL14 and AtSPL16, are not targets of miR156 in Arabidopsis. Among them, AtSPL7 can bind directly to the $\mathrm{Cu}$-response element $(\mathrm{CuRE})$ containing a core sequence of GTAC and is a regulator of $\mathrm{Cu}$ homeostasis in Arabidopsis [22]. AtSPL8 regulates pollen sac development [23], male fertility [24], GA biosynthesis and signaling [25]. AtSPL14 plays significant roles in plant development and sensitivity to fumonisin B1 [26]. Among the 19 rice SPLs, half are predominantly expressed in various young organs [27]. OsSPLs targeted by miR156 are involved in the development of flowers in rice. OsSPL14 regulated by miR156 also controls shoot branching in the vegetative stage $[8,28,29]$. In maize, liguleless1containing the SBP domain regulates ligule and auricle formation [30,31].

Populus trichocarpa is a model plant with whole genome sequence available [32]. A total of 352 miRNA precursors, including 12 for miR156, have been identified [33-39]. However, the regulation of miR156 in P. trichocarpa PtSPLs has not been analyzed. In our previous studies [40], 17 PtSPLs, which appeared to be full-length or partial sequence with at least 300 amino acids, were identified from the Populus genome assembly v1.1 (http:// genome.jgi-psf.org/Poptr1_1/Poptr1_1.home.html). They were named PtSPL1-PtSPL17, respectively, of which PtSPL3 and PtSPL4 had the highest similarities with AtSPL7 involved in $\mathrm{Cu}$ homeostasis [40]. In order to characterize the whole SPL gene family in P. trichocarpa, we searched the Populus genome assembly v1.1, v2.2 and v3.0 [32]. It resulted in the identification of 28 full-length PtSPLs. Gene structures, chromosomal locations, phylogenetic relationships, conserved protein motifs and expression patterns of all identified PtSPLs were systematically analyzed. MiR156-mediated posttranscriptional regulation of PtSPL genes was investigated. The results provide useful information for elucidating the biological functions of SPLs in P. trichocarpa.

\section{Results}

\section{Identification of $28 S P L$ genes in P. trichocarpa genome}

Analysis of the Populus genome assembly v1.1, v2.2 and v3.0 showed the existence of 28 full-length $S P L$ genes in the $P$. trichocarpa genome (Table 1). All of the deduced PtSPL proteins contained the conserved SBP domain. The theoretical $\mathrm{p} I$ of deduced PtSPL proteins ranged from 5.87 to 9.49. The length varied between 148 and 1044 amino acids. The molecular weight $(\mathrm{Mw})$ varied from 16.2 to $116.1 \mathrm{kDa}$ (Additional file 1). The distribution of $\mathrm{p} I$ is similar to AtSPLs (Additional file 2); however, the length and $\mathrm{Mw}$ of PtSPLs are larger than AtSPLs.
Mapping PtSPLs to the $P$. trhichocarpa genome showed that 28 PtSPLs were unevenly distributed on 14 chromosomes with four on Chr2, 3 on each of Chr1, Chr8, Chr10 and Chr14, 2 on each of Chr3, Chr11 and Chr15, and one on each of Chr4, Chr5, Chr7, Chr12, Chr16 and Chr18 (Figure 1). Relatively high densities of PtSPLs were observed in the top and bottom regions of Chr8, Chr10, Chr11 and Chr14, the top of Chr1, Chr4, and Chr16, and the bottom of Chr3, Chr5, Chr7, Chr12 and Chr18. Few are in the central regions of chromosomes. Moreover, 11 pair of PtSPLs (Ks $<1.0)$ were evolved from intrachromosomal duplication (Table 2), indicating the importance of gene duplication for PtSPL gene expansion.

\section{Phylogenetic analysis of SPLs in P. trichocarpa and Arabidopsis}

In order to investigate the evolutionary relationship between $P$. trichocarpa and A. thaliana SPL proteins, a neighbor-joining (NJ) phylogenetic tree was constructed for 28 PtSPLs and 16 AtSPLs using MEGA5.1. The reliability of branching was assessed by the bootstrap resampling method using 1,000 bootstrap replicates. Only nodes supported by bootstrap values $>50 \%$ are used for further analysis. The results showed that the 44 SPL proteins clustered into 6 groups (named G1-G6), each of which contained at least one AtSPL and one PtSPL (Figure 2). It is consistent with the results from SmSPLs in Salvia miltiorrhiza [41]. To further confirm that there are 6 groups of SPLs, we also constructed a phylogenetic tree for 28 PtSPLs, 16 AtSPLs, 18 rice OsSPLs and 15 SmSPLs. As shown in Additional file 3, the 77 SPLs also clustered into 6 groups. The difference between the two trees constructed (Figure 2, Additional file 3) is that PtSPL12, PtSPL13, PtSPL28 and AtSPL6 belonging to G1 in Figure 2 are included in G2 in Additional file 3. An intron was found in the SBP domain-encoding region of all SPL genes from P. trichocarpa and Arabidopsis (Figure 3); however, sequence feature analysis showed that the SBP domain of SPLs in G6 (AtSPL7, PtSPL3 and PtSPL4) were divergent from the other groups. The $\mathrm{N}$-terminal zinc finger of G6 SPLs has four cysteine residues in the SBP domain, while SPLs in the other groups mainly contain three cysteines and one histidine, indicating the diversification of plant SPL evolution. On the other hand, SPLs within a group have similar intron number, exon-intron structure, and coding sequence length. Consistently, the length, $\mathrm{Mw}$ and theoretical $\mathrm{p} I$ of deduced SPL proteins within a group are also similar, although they are divergent among groups. It suggests the conservation of plant SPLs in a group. Phylogenetic analysis showed that PtSPL3 and PtSPL4 had high homology with AtSPL7, an Arabidopsis SPL with the capability of binding CuREs in the MIR398 promoter in vitro 
Table 1 PtSPL gene names and gene model IDs in the Populus genome assembly v1.1, v2.2 and v3.0

\begin{tabular}{|c|c|c|c|}
\hline \multirow[t]{2}{*}{ Gene name } & \multicolumn{3}{|l|}{ Gene ID } \\
\hline & V1.1 & V2.2 & V3.0 \\
\hline PtSPL1 & GW1.X.791.1 & POPTR_0010s16370 & Potri.010G154000 \\
\hline PtSPL2 & FGENESH4_PM.C_LG_II000008 & POPTR_0002s00440 & Potri.002G002400 \\
\hline PtSPL3 & ESTEXT_FGENESH4_PM.C_LG_X0096 & POPTR_0010s02710 & Potri.010G026200 \\
\hline PtSPL4 & ESTEXT_FGENESH4_PM.C_LG_VIII0830 & POPTR_0008s20160 & Potri.008G197000 \\
\hline PtSPL5 $5^{\mathrm{a}}$ & $\begin{array}{l}\text { GRAIL3.0010027501 b }+ \text { GRAIIL3.0010027301 }^{\mathrm{b}}+ \\
\text { GRAIL3.0010027401 }^{\mathrm{b}}\end{array}$ & POPTR_0008s09810 & Potri.008G098600 \\
\hline PtSPL6 & ESTEXT_GENEWISE1_V1.C_LG_XIV2145 b + GW1.XIV.2149.1 b & POPTR_0014s10960 & Potri.014G114300 \\
\hline PtSPL7 & GRAIL3.0050015101 ${ }^{\mathrm{b}}+\mathrm{GW} 1.8978 .5 .1^{\mathrm{b}}+\mathrm{GW} 1.11 .489 .1^{\mathrm{b}}$ & POPTR_0002s18970 & Potri.002G188700 \\
\hline PtSPL8 & FGENESH4_PG.C_LG_II001303 & POPTR_0002s14330 & Potri.002G142400 \\
\hline PtSPL9 & EUGENE3.00051637 + EUGENE3.00051638 ${ }^{\mathrm{b}}$ & POPTR_0005s28010 & Potri.005G258700 \\
\hline PtSPL11 & GRAIL3.0047015901 b & POPTR_0003s17120 & Potri.003G172600 \\
\hline PtSPL12 & GRAIL3.0010026901 & POPTR_0008s09750 & Potri.008G097900 \\
\hline PtSPL13 & FGENESH4_PG.C_LG_X001404 & POPTR_0010s16400 & Potri.010G154300 \\
\hline PtSPL14 & ESTEXT_GENEWISE1_V1.C_LG_XV2187 & POPTR_0015s11100 & Potri.015G098900 \\
\hline PtSPL15 & EUGENE3.00120942 & POPTR_0012s10260 & Potri.012G100700 \\
\hline PtSPL16 & ESTEXT_GENEWISE1_V1.C_1240186 & POPTR_0011s05480 & Potri.011G055900 \\
\hline PtSPL17 $7^{\mathrm{a}}$ & EUGENE3.00160416 & $\begin{array}{l}\text { POPTR_0016s04880 }+ \\
\text { POPTR_0016s04890 }\end{array}$ & Potri.016G048500 \\
\hline PtSPL18 & GW1..1.7783.1 ${ }^{\mathrm{b}}$ & POPTR_0001s13630 & Potri.001G058600 \\
\hline PtSPL19 & GW1.1.7690.1 ${ }^{\mathrm{b}}$ & POPTR_0001s13890 & Potri.001G055900 \\
\hline PtSPL20 & GW1.107.39.1 & POPTR_0001s40870 & Potri.001G398200 \\
\hline PtSPL21 & GW1.II.3778.1 b & POPTR_0002s14320 & Potri.002G142200 \\
\hline PtSPL22 & GW1.III.2396.1 & POPTR_0003s16780 & Potri.003G169400 \\
\hline PtSPL23 & GW1.IV.3037. $1^{\mathrm{b}}$ & POPTR_0004s04630 & Potri.004G046700 \\
\hline PtSPL24 & GW1.VII.548.1 $1^{\mathrm{b}}$ & POPTR_0007s01030 & Potri.007G138800 \\
\hline PtSPL25 & GW1.XI.3794. $1^{\mathrm{b}}$ & POPTR_0011s11770 & Potri.011G116800 d \\
\hline PtSPL26 & GW1.40.81.1 ${ }^{\mathrm{b}}$ & POPTR_0014s05680 & Potri.014G057700 \\
\hline PtSPL27 & GW1.40.76.1 ${ }^{\mathrm{b}}$ & POPTR_0014s05690 & Potri.014G057800 \\
\hline PtSPL28 & GW1.129.152.1 & POPTR_0015s07140 & Potri.015G060400 \\
\hline PtSPL29 & GW1.164.76.1 ${ }^{b}$ & POPTR_0018s14680 & Potri.018G149900 \\
\hline
\end{tabular}

${ }^{\mathrm{a}}$ Genes are split into 2 or 3 gene models in v1.1 or v2.2;

${ }^{\mathrm{b}} \mathrm{Gene}$ models with partial sequence;

'The gene model includes 7 additional amino acids at the $\mathrm{N}$-terminal compared with the model in v1.1.

${ }^{\mathrm{d}}$ The gene model includes 49 additional amino acids at the N-terminal compared with the model in v2.2.

and involved in response to copper deficiency in Arabidopsis [22]. It is consistent with our previous results for PtSPLs [40]. Based on the phylogenetic tree, PtSPL3 and AtSPL7 are very likely to be orthologous proteins (Figure 2). Additionally, 5 pairs of AtSPLs and 11 pairs of PtSPLs seem to be paralogous proteins (Figure 2). It includes AtSPL9/15, AtSPL10/11, PtSPL8/27, PtSPL12/13 and PtSPL11/19 belonging to G1, PtSPL18/22 and PtSPL14/15 from G2, PtSPL21/26 belonging to G3, AtSPL14/16, AtSPL1/12, PtSPL2/9, PtSPL1/5 and PtSPL6/ 7 included in G4, and AtSPL3/4, PtSPL16/23 and PtSPL20/ 25 clustering in G5. About $62.5 \%$ of the 16 AtSPLs and
$78.5 \%$ of the 28 PtSPLs exist as paralogous pairs. It suggested that the expansion of SPL genes occurred after separation of paralogous genes. The results from paralogous pair identification were consistent with segmental duplications in the $P$. trichocarpa genome (http://chibba.agtec.uga. edu/duplication/) [32], suggesting the origination of paralogous PtSPLs from segmental duplication. Prediction of potential age of tandem duplication events using synonymous substitutions (Ks) values showed that the segmental duplication events for PtSPLs appeared to occur in 9-21 mya (Table 2). It is consistent with the age of $P$. trichocarpa genome duplication events [32]. 


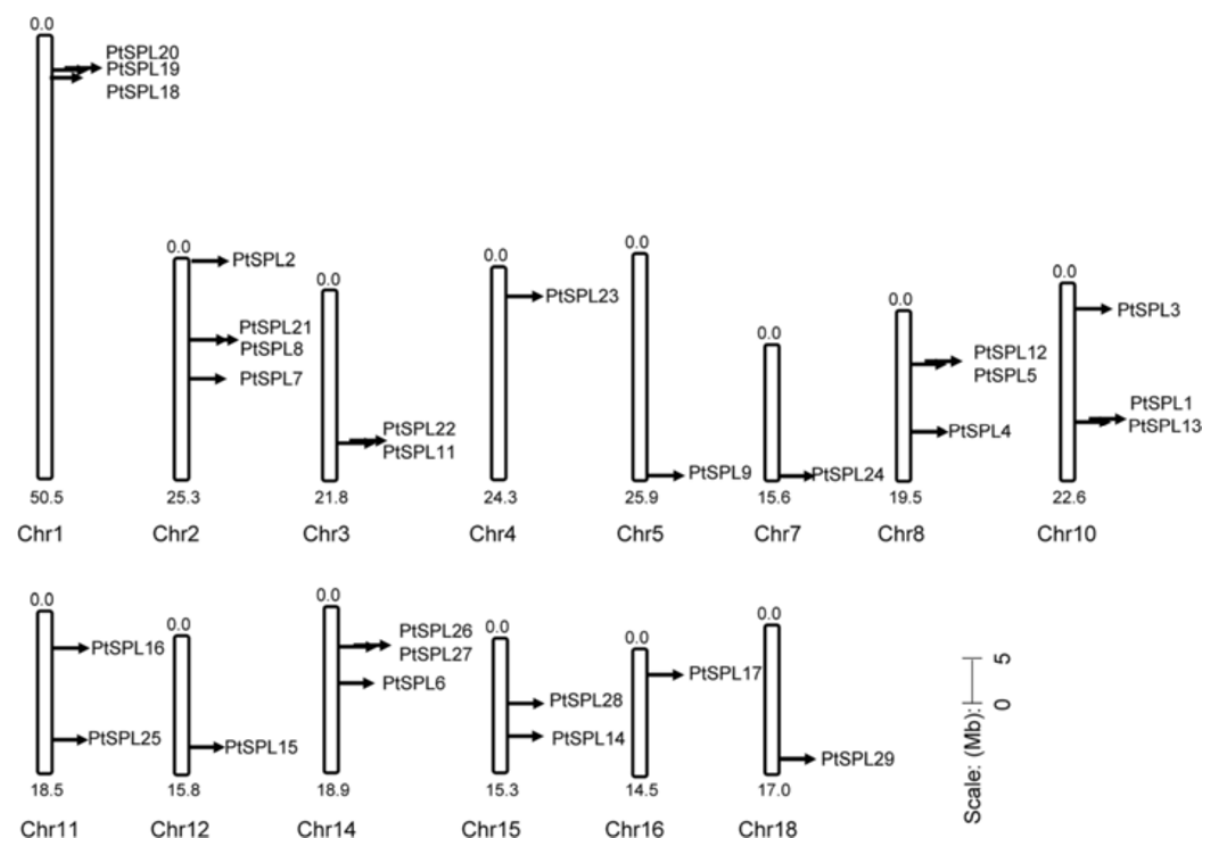

Figure 1 Chromosomal location of PtSPL genes. Scale represents a 5 Mb chromosomal distance.

\section{Comparative analysis of PtSPL and AtSPL gene structures}

Gene structure analysis showed that the number of introns in the coding region of 28 PtSPL genes varied from 1 to 10 . The number of PtSPLs with $1,2,3,4,9$ and 10 introns is five, ten, four, one, six, and two, respectively (Figure 3, Additional file 1). Similarly, the intron number of 16 AtSPLs varies between 1 and 9 (Additional file 2). The pattern of intron distribution in PtSPLs is quite similar to AtSPLs with the majority to be 2 and 9 introns, followed by 1 and 3 (Figure 3, Additional files 1 and 2) [41]. In addition, the position of intron in the SBP domain is highly conserved. It locates in the codon for the 48th amino acid of SBP domain (Additional

Table 2 Estimated age of the duplication events for PtSPL paralogous genes

\begin{tabular}{llc}
\hline Paralogous genes & Ks & Estimated time (mya) \\
\hline PtSPL19(Chr1)/PtSPL11(Chr3) & 0.16 & 9 \\
PtSPL21(Chr2)/PtSPL26(Chr14) & 0.31 & 17 \\
PtSPL8(Chr2)/PtSPL27(Chr14) & 0.29 & 16 \\
PtSPL2(Chr2)/PtSPL9(Chr5) & 0.27 & 15 \\
PtSPL12(Chr8)/PtSPL13(Chr10) & 0.24 & 13 \\
PtSPL5(Chr8)/PtSPL1(Chr10) & 0.22 & 12 \\
PtSPL18(Chr1)/PtSPL22(Chr3) & 0.17 & 9 \\
PtSPL23(Chr 4)/PtSPL16(Chr11) & 0.22 & 12 \\
PtSPL20(Chr1)/PtSPL25(Chr11) & 0.39 & 21 \\
PtSPL7 (Chr2)/ PtSPL6 (Chr14) & 0.25 & 13 \\
PtSPL15(Chr12)/PtSPL14(Chr15) & 0.30 & 16 \\
\hline
\end{tabular}

file 4). These results suggest the conservation of exonintron structures between PtSPLs and AtSPLs.

The length of introns varies significantly among PtSPL genes, such as those in G1, G2 and G5 (Figure 3). We analyzed the internal exons and introns of PtSPLs and AtSPLs. The results showed that the exons of PtSPLs had a size from 43 to 884 bp with an average of $314 \mathrm{bp}$, which is slightly greater than $293 \mathrm{bp}$ of the average length of AtSPL exons. Approximately 59\% of PtSPL exons and $63 \%$ of $A t S P L$ exons have a size below $300 \mathrm{bp}$ and $71 \%$ and $70 \%$ of exons are between 60 and 160 bp in PtSPLs and AtSPLs, respectively (Figure 4). Although the size distribution of PtSPLs exons is similarity with AtSPL exons, intron size distribution is more variable, ranging from $30 \mathrm{bp}$ to $3.0 \mathrm{~kb}$. There are $6 \mathrm{PtSPL}$ introns (5\%) with sizes $>1.5 \mathrm{~kb}$; however, no such introns exist in AtSPLs. About 55\% of PtSPLs have sizes below $300 \mathrm{bp}$ and $56 \%$ of introns are between 60 and $160 \mathrm{bp}$; however, the majority of AtSPLs (94\%) have sizes below $300 \mathrm{bp}$. The average size of PtSPL introns is $476 \mathrm{bp}$, which is much greater than $120 \mathrm{bp}$ of AtSPLs. These results suggest the difference of exon and intron size distribution between PtSPLs and AtSPLs.

\section{Identification of $\mathbf{2 5}$ conserved motifs}

Conserved domains of PtSPLs were analyzed using Pfam (http://pfam.sanger.ac.uk) and by BLAST analysis of protein sequences against the Conserved Domain Database (CDD, http://www.ncbi.nlm.nih.gov/Structure/cdd/wrpsb.cgi). The results showed that all of the 28 PtSPLs and 16 AtSPLs contained a SBP domain with about 78 amino acid residues 


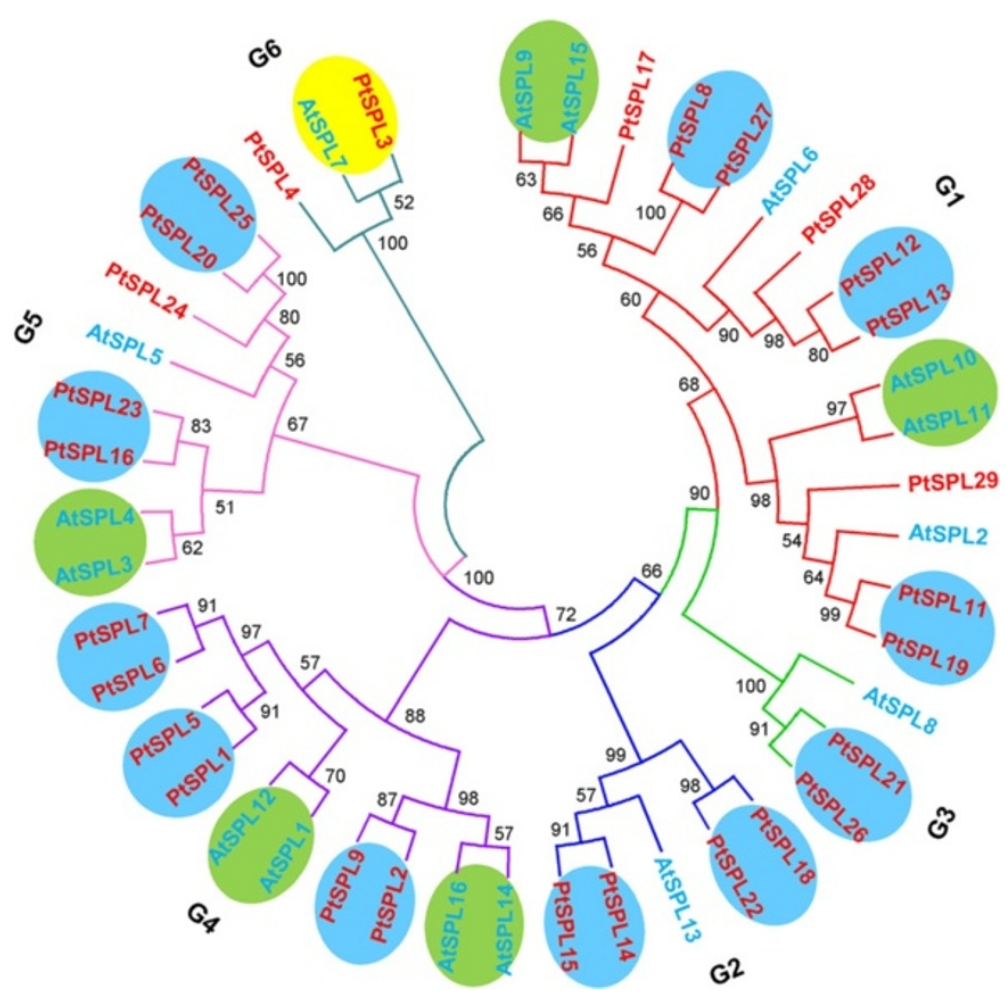

Figure 2 Neighbor-joining (NJ) phylogenetic tree for 44 SPLs in P. trichocarpa and Arabidopsis. The groups of homologous genes identified and bootstrap values are shown. The reliability of branching was assessed by the bootstrap re-sampling method using 1,000 bootstrap replicates. Bootstrap values are shown below nodes.

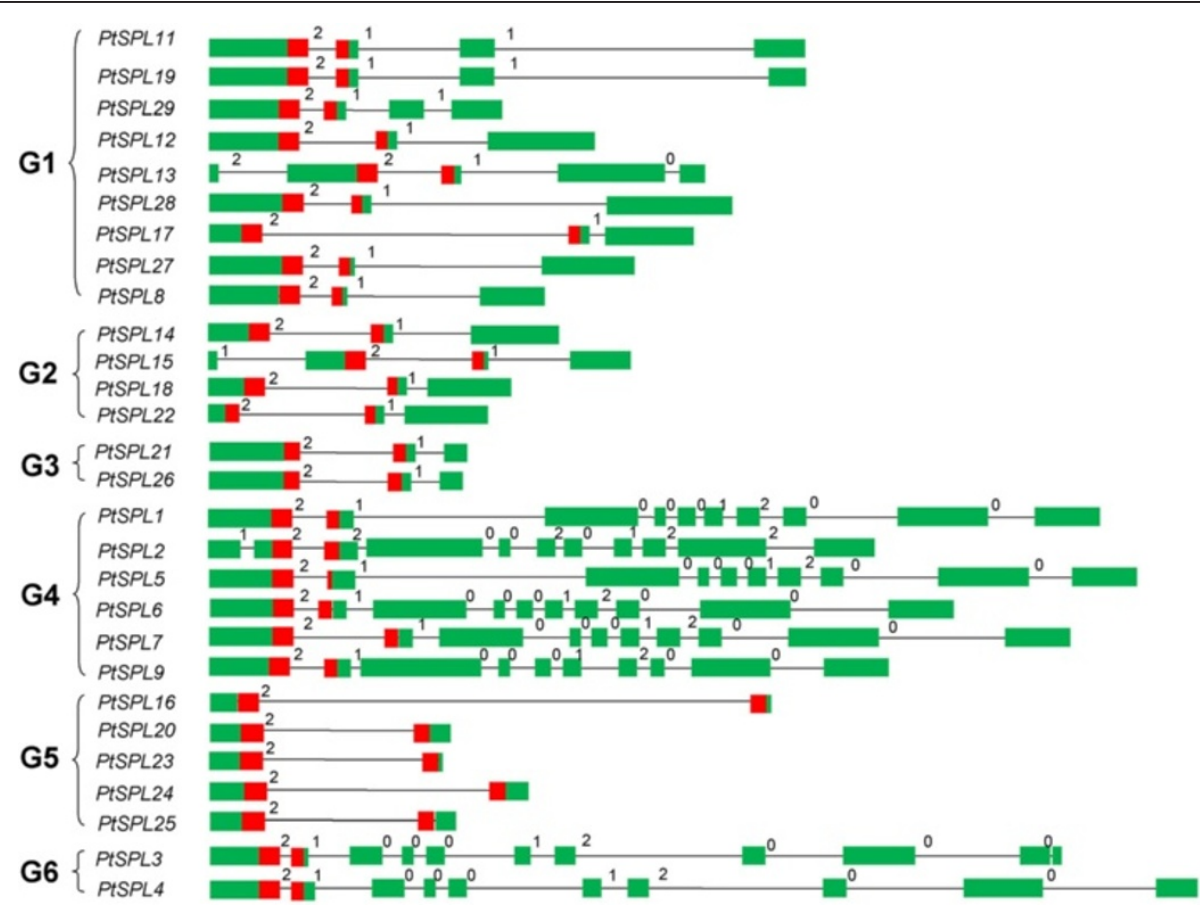

Figure 3 Exon-intron structures of PtSPLs. Introns are represented by lines. Exons are indicated by green boxes. The SBP domains are shown in red boxes. Intron phases are shown by 0,1 and 2. 


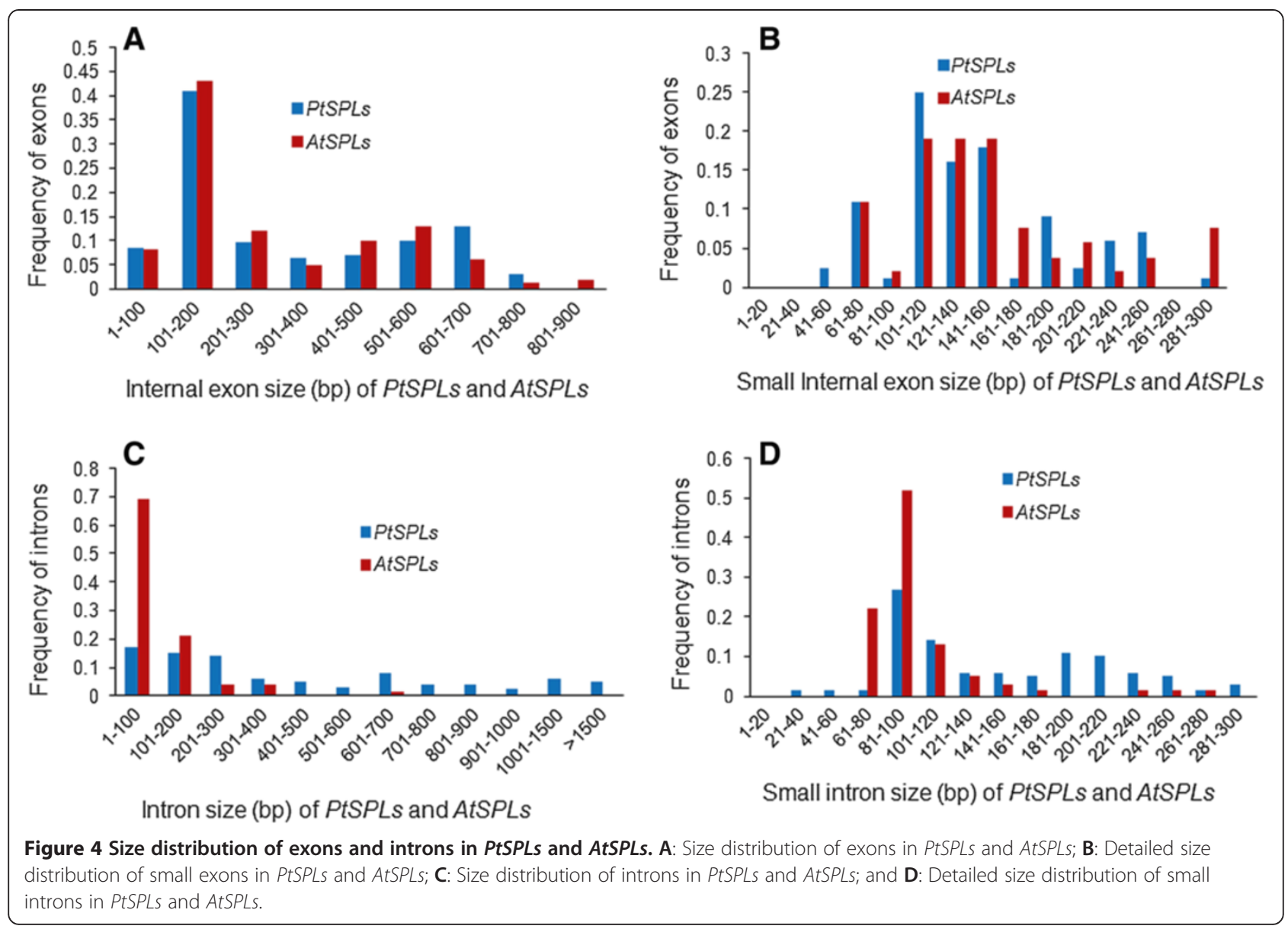

in length (Figure 5). It is not surprising given that the SBP domain was used for PtSPL identification. Sequence analysis of SBP domains revealed that the conserved zincbinding sites, $\mathrm{Zn} 1$ and $\mathrm{Zn} 2$, also existed in the SBP domain of PtSPLs (Figure 5). Zn1 is Cys3His-type (CCCH-type) in G1-G5 SPLs (Figure 5A); however, the His residue in Zn1 is replaced by a Cys residue in G6, which results in the signature sequence of G6 SPLs to be CCCC (Figure 5B). Unlike $\mathrm{Zn} 1$, the signature sequence $(\mathrm{C} 2 \mathrm{HC})$ of $\mathrm{Zn} 2$ is highly conserved in all SPLs analyzed. In addition to $\mathrm{Zn} 1$ and $\mathrm{Zn} 2$, the SBP domain contains a conserved nuclear location signal (NLS) in the C-terminus of SBP domains (Figure 5). The conservation of SBP domains between PtSPLs and AtSPLs indicates that the domain organization has been established in ancient plants. Moreover, six PtSPLs (PtSPL1, PtSPL2, PtSPL5, PtSPL6, PtSPL7 and PtSPL9) belonging to G4 contain an ANK or Ank-2 domain with three or four ankyrin repeats (Additional file 5), which are involved in protein-protein interaction [42]. It is consistent with previous results from AtSPLs and SmSPLs [41].

In addition to the conserved domains, other conserved motifs could also be important for the function of SPLs $[27,43]$. We searched conserved motifs using MEME and applied an e-value cut off of $1 \mathrm{e}^{-10}$ to the recognition. It resulted in the identification of 25 motifs for 28 PtSPLs (Figure 6, Table 3). The majority of motifs identified are conserved between PtSPLs and AtSPLs [41], while three, including motifs 11, 19 and 23, are specific to PtSPLs. It indicates the conservation and diversity of PtSPLs and AtSPLs. The number of motifs in each SPL varies from 1 to 16 (Figure 6). Motif 1 is actually the SBP domain. Consistently, it exists in all SPLs analyzed. Motif 14 existed in G1 and G2 SPLs contains the target gene sequence of miR156, indicating the posttranscriptional regulation of G1 and G2 SPLs by miR156. In addition to motifs 1 and 14, several motifs widely exist in two SPL groups, such as motif 12 found in G1 and G2, motifs 2, 4, 5, 6, 15 and 16 existing in G4 and G6 (Figure 6), indicating the importance of these motifs. We also found several motifs to be group-unique, such as motif 24 specifically existing in G6 SPLs and motifs 7, 9, 10 and 18 specific to G4 (Figure 6). These group-unique motifs could be important for specific roles of SPLs in the group. Moreover, PtSPLs and AtSPLs [41] within a group share similar motif (s), indicating they probably play similar roles in plant growth and development. 

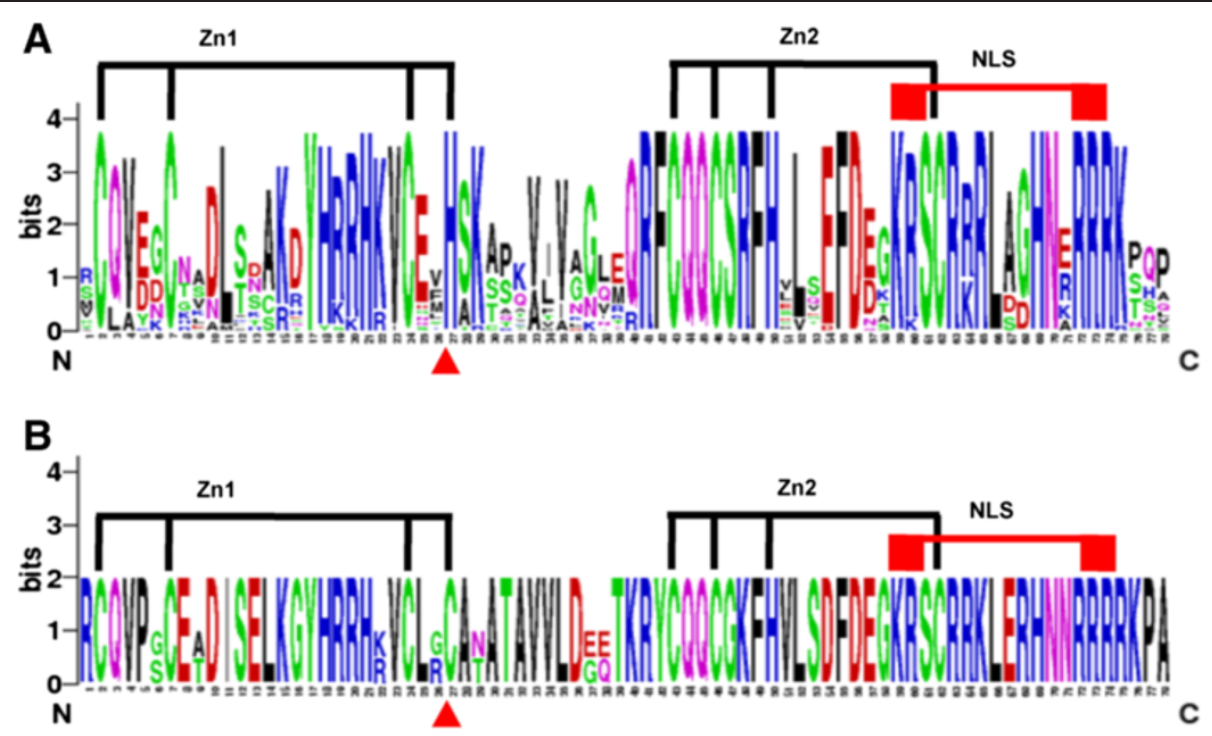

Figure 5 Sequence logo of the SBP domain of PtSPLs. A: Sequence logo of the SBP domain of PtSPLs in G1-G5; B: Sequence logo of the SBP domain of PtSPLs in G6. Two conserved Zn-finger structures and the NLS are indicated.

\section{Expression patterns of SPLs in $P$. trichocarpa}

The expression pattern of a gene is often correlated with its function. In order to preliminarily elucidate the roles of PtSPLs in P. trichocarpa development, we first searched PopGenIE for gene expression data from microarray analysis [44]. Except for PtSPL17, the expression levels of 27 PtSPLs in roots, stems, young leaves and mature leaves were obtained (Figure 7). Next, we examined the relative expression levels of 28 PtSPLs in young leaves, mature leaves, young stems, young roots and tissues from developing secondary xylem and phloem from the 4th-6th and 12th-25th internodes of one-year-old $P$. trichocarpa plants using the quantitative real-time RT-PCR method (Figure 8). The results showed that qRT-PCR data was generally consistent with microarray data for relative expression of PtSPLs in roots, stems, young leaves and mature leaves (Figures 7 and 8). Although all PtSPLs were expressed in at least one of the tissues examined, differential expression was observed. Many putative paralogous genes, such as PtSPL18/22 in G2, PtSPL21/26 in G3, PtSPL2/9, PtSPL1/ 5 and PtSPL6/7 in G4 and PtSPL16/23 belonging to G5, show similar expression patterns, suggesting redundant roles of these PtSPL gene pairs. However, the expression patterns of few gene pairs, including PtSPL12/13 in G1, and PtSPL14/15 belonging to G2 are distinct. It indicates these PtSPLs may play different roles in P. trichocarpa development, although they are paralogous genes.

MiR156-mediated posttranscriptional regulation of PtSPLs It has been shown that 10 AtSPLs are regulated by miR156 [11]. The complementary sites of miR156 are in the coding regions or 3' UTRs of AtSPLs. In order to know miR156-medicated posttranscriptional regulation of PtSPLs, we searched coding regions and 3' UTRs of all PtSPLs for targets of $P$. trichocarpa miR156amiR156j on the psRNATarget server using default parameters [45]. The results showed that 18 PtSPLs were potential targets of miR156 (Figures 9 and 10). MiR156targeting sites in 13 PtSPLs belonging to G1 and G2 locate in the last exon and encode the conserved peptide ALSLLS. The target sites for other 5 PtSPLs belonging to G5 locate in the 3' UTRs close to the stop codons (Figure 10). Consistently, AtPSLs clustering in G1, G2 and G5 are targets of miR156 in Arabidopsis. It suggests that miR156-mediated posttranscriptional regulation of SPLs is conserved in P. trichocarpa and Arabidopsis.

\section{Discussion}

SPLs are plant-specific transcription factors containing a highly conserved SBP (SQUAMOSA PROMOTER BINDING PROTEIN) domain. It can specifically bind to the promoters of floral meristem identity gene SQUA$M O S A$ and its orthologous genes and plays important regulatory roles in plant growth and development [46-49]. The genes encoding SPLs have been identified from various plant species, such as Arabidopsis [2,10,23,26], maize [30], Antirrhinum majus [3], rice [50], silver birch [51], and S. miltiorrhiza [41]. SPL genes exist as a large gene family in plants. The number of SPLs in Arabidopsis, rice, P. patens, maize and tomato is $16,19,13,31$ and 15, respectively [4-9]. Availability of the whole genome sequence allows us to perform genome-wide identification of SPLS in P. trichocarpa. Analysis of three versions of the annotated $P$. trichocarpa genome showed the existence of 28 full-length 


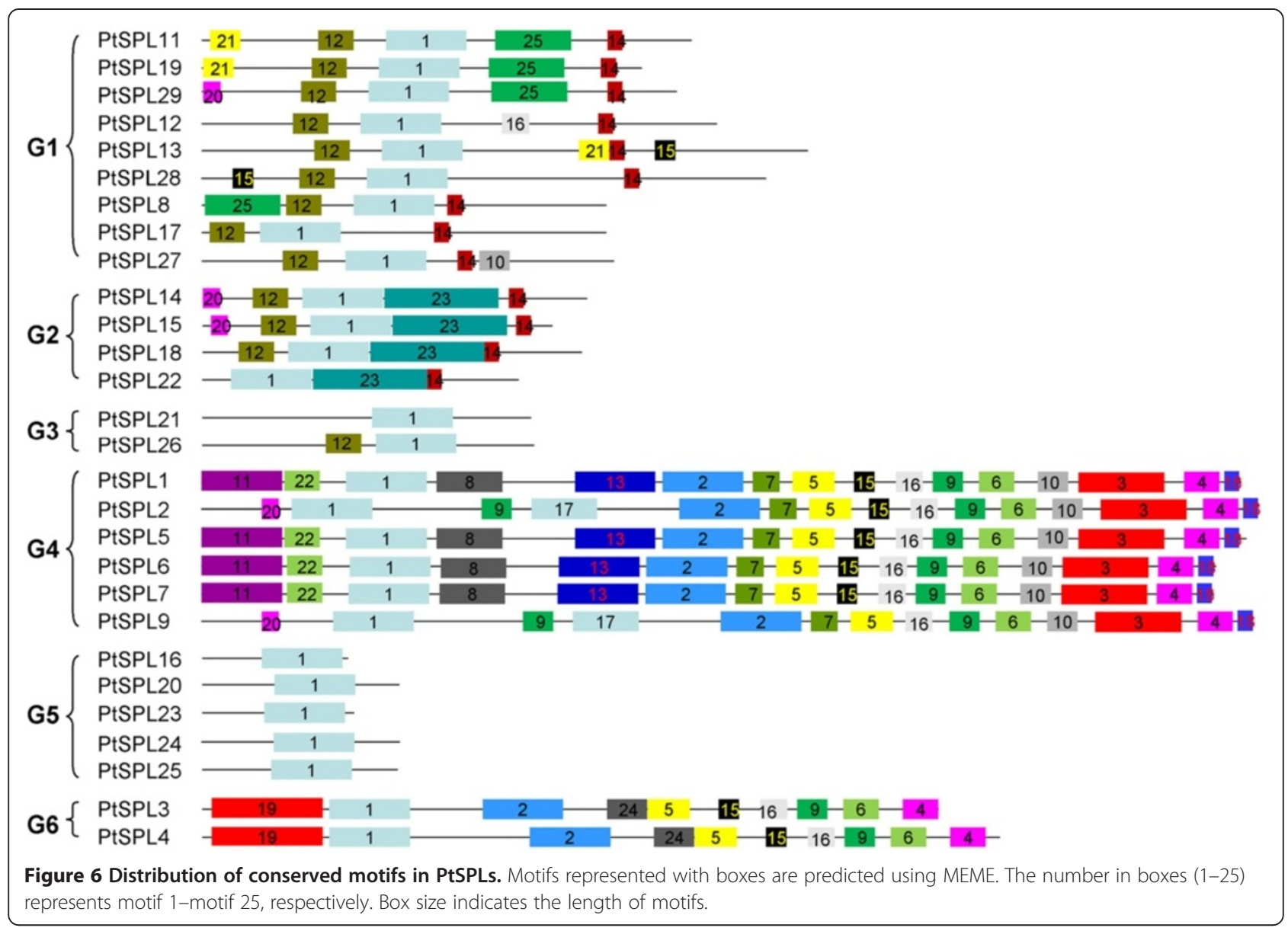

PtSPLs, which distribute on 14 chromosomes. It is the first attempt to analyze the PtSPL gene family. The results provide a basis for elucidating the functions of SPLS in P. trichocarpa, a model forest tree.

The number of SPL genes in P. trichocarpa is much greater than that in Arabidopsis, rice, P. patens and tomato, although it is similar to the number of maize SPLs [4-9]. Sequence homologous analysis suggests that gene duplication plays an important role in $S P L$ gene expansion in $P$. trichocarpa. A total of 11 pairs of intrachromosome-duplicated PtSPLs were identified in this study. All of them clustered together in the phylogenetic tree (Figure 2). It is consistent with previous findings for generation and maintenance of gene families in other organisms, such as mouse, human and Arabidopsis [52,53]. Actually, gene duplication has been reported for many plant transcription factor gene families, such as $M Y B, A P 2, M A D S$ and so on [54-56] and duplicated SPL gene pairs have been identified in Arabidopsis (AtSPL10/11, AtSPL4/5 and AtSPL1/12) and rice (OsSPL2/ 19, OsSPL3/12, OsSPL4/11, OsSPL5/10 and OsSPL16/18) [57-61]. However, the number of homologous PtSPL gene pairs is obviously greater than that in Arabidopsis and rice, indicating that more segment duplication events happened in Populus and most SPL genes in Arabidopsis and Populus expanded in a species-specific manner [62-64].

Comparative analysis of $P$. trichocarpa PtSPLs and Arabidopsis AtSPLs revealed many conserved sequence features. For instance, all of the deduced proteins contain the highly conserved SBP domain with about 78 amino acid residues. The intron position and intron phase in the SBP-domain-encoding regions are also conserved among all SPL genes in P. trichocarpa and Arabidopsis, indicating that plant SPL genes originate from a common ancestor. Based on the neighbor-joining (NJ) phylogenetic tree constructed using MEGA 5.1., 44 SPL proteins from P. trichocarpa and Arabidopsis were found to cluster into 6 groups. Each group includes at least a PtSPL and one AtSPL. The intron number and intron phase are similar for PtSPLs and AtSPLs within a group. The results suggest the conservation between $P$. trichocarpa PtSPLs and Arabidopsis AtSPLs.

It has been shown that AtSPLs play significant regulatory roles in a variety of developmental processes in Arabidopsis. For instance, morphological traits of cauline leaves and flowers are regulated by AtSPL2, AtSPL10 and AtSPL11 [19]. Juvenile-to-adult growth phase transition and leaf initiation rate are controlled by the 


\begin{tabular}{|c|c|c|}
\hline Motif & E-value & Consensus sequence \\
\hline 1 & $1.0 e-2549$ & CQVEGCNADLSSAKDYHRRHKVCEVHSKAPKVIVAGLEQRFCQQCSRFHLLSEFDEGKRSCRRRLAGHNERRRKPQPD \\
\hline 2 & $3.3 e-516$ & SDQPSSSSSSGDAQCRTGRIVFKLFDKDPNDFPGTLRTQILDWLSHSTDMESYIRPGCILTIYLAMPEAAWEELCCDLG \\
\hline 3 & $6.2 \mathrm{e}-387$ & LFRPDVAGPAGLTPLHIAACKDGSEDVLDALTEDPGEVGISAWKNARDATGFTPYARLRGHHSYIHLVQRKLADKRNGQVSWI \\
\hline 4 & 3.6e-174 & ASRSLLYRPAMLSMVAIAAVCVALLFKSCPEVLY \\
\hline 5 & $9.0 \mathrm{e}-136$ & VEAGEETEFWKGRNLYQPGTRLLCAVEGKYLVQETTQALMD \\
\hline 6 & $7.4 \mathrm{e}-135$ & FPLRRFKFLLEFSMDRDWCAWRKLLDMLVEGNVCRD \\
\hline 7 & $5.6 e-100$ & FWRTGWFYVRVQNQLAFHKNGQWLDTSL \\
\hline 8 & $3.0 \mathrm{e}-085$ & GGSMNDDQGYLLTSILSNLHSNRSDQTKDQDLLSHLLRSLASHAGEHNGRNLFGLLQGPRGL \\
\hline 9 & $1.8 \mathrm{e}-095$ & EGMPSKEQALDFLNEIGWLLHRSDLKSRL \\
\hline 10 & 4.8e-088 & SSLEALSEMGLLHRAVRRNSRKMVELLLR \\
\hline 11 & 7.4e-085 & MEARFGGESHHFYAPVPSDLKAVGKRGLEWDLNDWKWDGDLFIASPLNPVPSDCRSRQFFPTGPGLGEKAGGNNSNSSCS \\
\hline 12 & $3.2 \mathrm{e}-077$ & STSLGASxSSGESLLGLKLGKRIYFEDAxGXNNxK \\
\hline 13 & $3.5 e-070$ & FSIPNNFAAKSEEPEATAGQIKLNFDLNDIYDDSDDGIEDIERSHAPVNAGMGSFDCPLMVQQDSHKSSPPHTSGNSDS \\
\hline 14 & $1.3 e-052$ & ASDSDCALSLLSSQS \\
\hline 15 & $8.9 e-057$ & NFSCSXPNLLGRGFIEVED \\
\hline 16 & $9.8 \mathrm{e}-064$ & PFIIADADVCSEIRILEQEFD \\
\hline 17 & 8.7e-053 & GERISSCNESPSEDSDSQGQDSRPNLPLQLFSSSPENESRPKVASSRKYFSSASSNPIEDRSPSSSP \\
\hline 18 & $1.8 \mathrm{e}-039$ & PFRWELLDYGT \\
\hline 19 & 5.1e-035 & QHDGDMEIHLPPITTDWDWGDILDFAVDDQFPLSFDTPGDLTQPIDNPTPEIESQQLEAPVPDRVRKRDPRLTCSNFLAGIVPCACPEMDELLLEEEAALPGKKRVRVARAG \\
\hline 20 & 2.8e-033 & DDWNLKAWDWDGDEFEA \\
\hline 21 & $8.5 e-032$ & MDCNGKPHLQWDWENLIMFNAITTENSKK \\
\hline 22 & 2.7e-030 & DEDNLGDEKGKRELEKRRRWFIDDDNLND \\
\hline 23 & $3.3 e-028$ & VNSARIFSNQGTRYLHFGSSQIFSTSAMNAAWTGAAKAERDPMLNTSQSSMNFDGRKNLFPGSLSPNYKEGKQFPFLQGTSSTIPGDSIHLDANSTLGNSQKMFSDGLNR \\
\hline 24 & $3.4 \mathrm{e}-027$ & KGRMRVYLNNMIFNVTKDGHSVMKVNVKGHAPRLHYVHPTC \\
\hline 25 & $5.9 e-025$ & DERQQMSHAWDKAPLVHARPNANLTWEGTSISKFTITKDYIAKPAEIGGNDGQFHLPGFDLTNGIATQHHHKSN \\
\hline
\end{tabular}




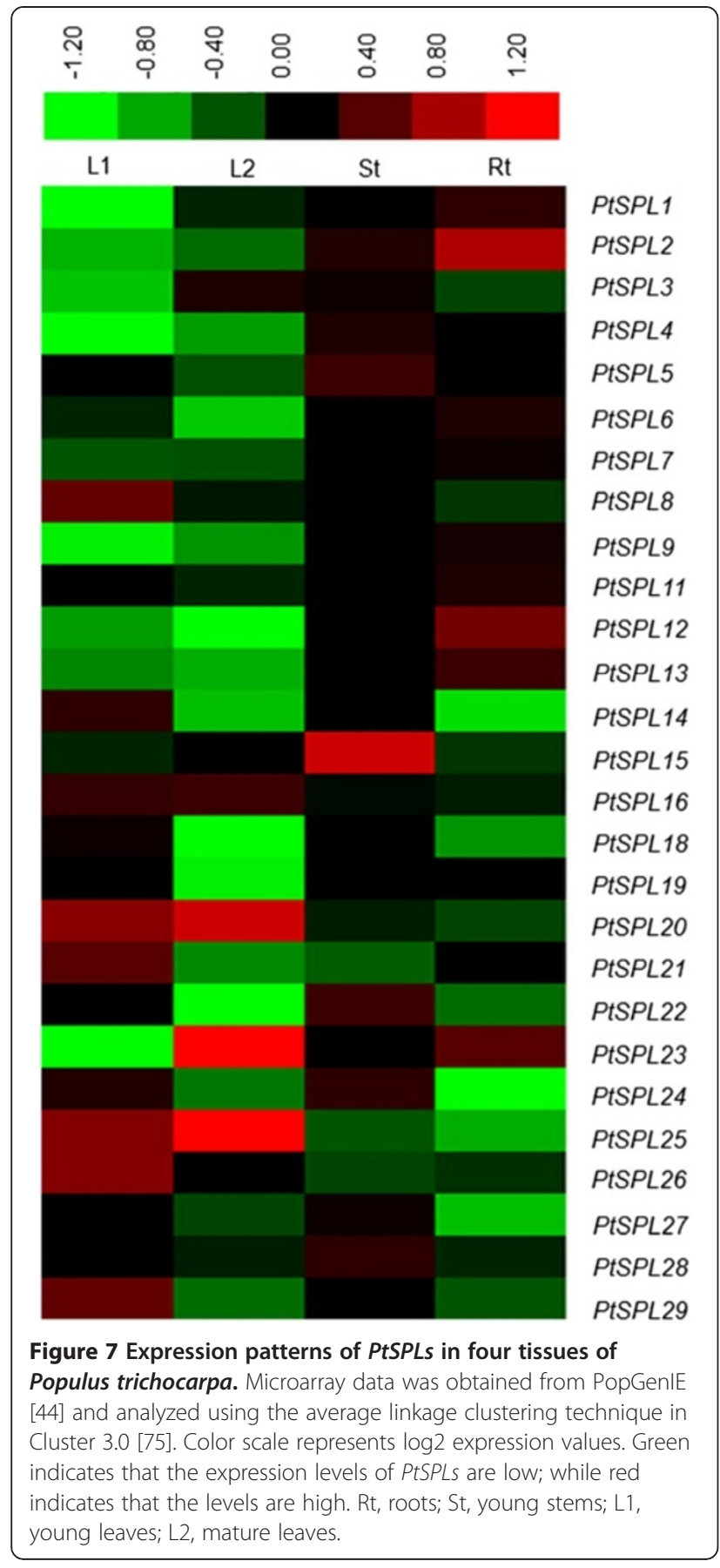

redundant action of AtSPL9 and AtSPL15 [21]. Pollen sac development, male fertility and GA biosynthesis and signaling are regulated by AtSPL8, a member of G3 [23-25]. $\mathrm{Cu}$ homeostasis in Arabidopsis is regulated by the member of group 6, AtSPL7 [22]. In this study, we found that many motifs were unique to or mainly existed in a group of SPLs. It is consistent with the redundant roles of AtSPLs in a group and indicates that the members of PtSPLs in the same group may play similar roles as their Arabidopsis counterparts. The function of SPLs in different groups could be functionally distinct. On the other hand, three PtSPL-specific motifs, including motifs 11, 19 and 23, were identified, suggesting that some PtSPLs may play species-specific roles. Consistently, most of paralogous PtSPL gene pairs in the same group show similar expression patterns, whereas a few of them exhibit differential patterns. The results indicate subfunctionalisation and neofunctionalisation of SPLs within a plant species and among different species.

MiR156-medicated posttranscriptional regulation is important for the function of a subset of SPLs [11,41,65]. Target prediction showed that all PtSPLs in groups 1, 2 and 5 were regulated by miR156. The complementary sites of miR156 locate in the coding region of G1 and G2 SPLs, whereas it locates in 3' UTR of G5 SPLs. It is consistent with the results from Arabidopsis SPLS and suggests the conservation of miR156-mediated posttranscriptional regulation in plants.

\section{Conclusion}

In this study, a total of 28 full-length SPLs were identified from the whole genome sequence of $P$. trichocarpa. Through a comprehensive analysis of gene structures, phylogenetic relationships, chromosomal locations, conserved motifs, expression patterns and miR156-mediated posttranscriptional regulation, the PtSPL gene family was characterized and compared with SPLs in Arabidopsis. The results showed that 28 PtSPLs and 16 AtSPLs clustered into 6 groups. Many PtSPLs and AtSPLs within a group are highly conserved in sequence features, gene structures, motifs, expression patterns and posttranscriptional regulation, suggesting the conservation of plant SPLs within a group. However, significant differences were observed for SPLS among groups. In addition, various motifs were identified in PtSPLs but not in AtSPLs. It suggests the diversity of plant SPLs. The results provide useful information for elucidating the functions of SPLS in P. trichocarpa.

\section{Methods}

\section{Identification of PtSPL genes}

The nucleotide sequences and deduced amino acid sequences of 16 known SPL genes in Arabidopsis [2,4] were obtained from the TAIR database (http://www.arabidopsis.org) (Additional file 2). The SBP domain of AtSPLs was identified using Pfam (http://pfam.sanger.ac. uk). BLAST search of PtSPLs against Populus trichocarpa v1.1, v2.2 and v3.0 was carried out using AtSPL SBP as the query sequences [32] (http://genome.jgi-psf.org/ Poptr1_1/Poptr1_1.home.html,http://www.phytozome.net/ poplar.php\#B). An e-value cut off of $1 \mathrm{e}^{-5}$ was applied to the recognition. We also searched the databases for SBP using the keywords search tool on the web servers. Protein 


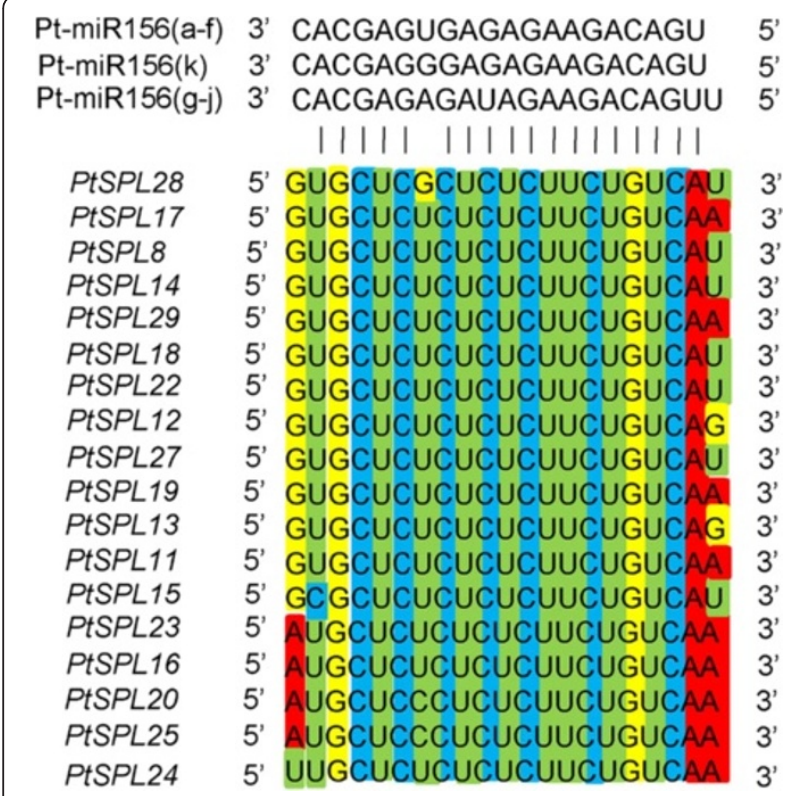

Figure 9 Sequence alignment of $P$. trichocarpa miR156amiR156j with their complementary sequence in coding regions and 3' UTRs of 18 PtSPLs. sequences retrieved from Populus trichocarpa v1.1, v2.2 and v3.0 were then aligned and combined based on sequence identities.

\section{Chromosome location and sequence feature analyses}

Chromosome locations of PtSPL genes were determined by BLAST analysis of PtSPLs against Populus trichocarpa v3.0 (http://www.phytozome.net/poplar.php\#B). Paralogous gene pairs were analyzed on the Plant Genome Duplication Database (PGDD) server (http://chibba.agtec. uga.edu/duplication/index/locus) with display range for $100 \mathrm{~kb}$. The approximate date of the duplication events was calculated using $\mathrm{T}=\mathrm{Ks} / 2 \lambda$ by assuming clock-like rates $(\lambda)$ in Populus for $9.0 \times 10^{-9}[32,57,66]$. Synonymous substitutions (Ks) values of paralogous gene pairs were calculated using DnaSP [67]. The theoretical isoelectric point $(\mathrm{p} I)$ and molecular weight $(\mathrm{Mw})$ were predicted using the Compute $\mathrm{pI} / \mathrm{Mw}$ tool on the ExPASy server (http://web.expasy.org/compute_pi/) [68]. The intron/exon structure of SPL genes was predicted with the Gene Structure Display Server (http://gsds.cbi.pku.edu. cn/chinese.php) [69].

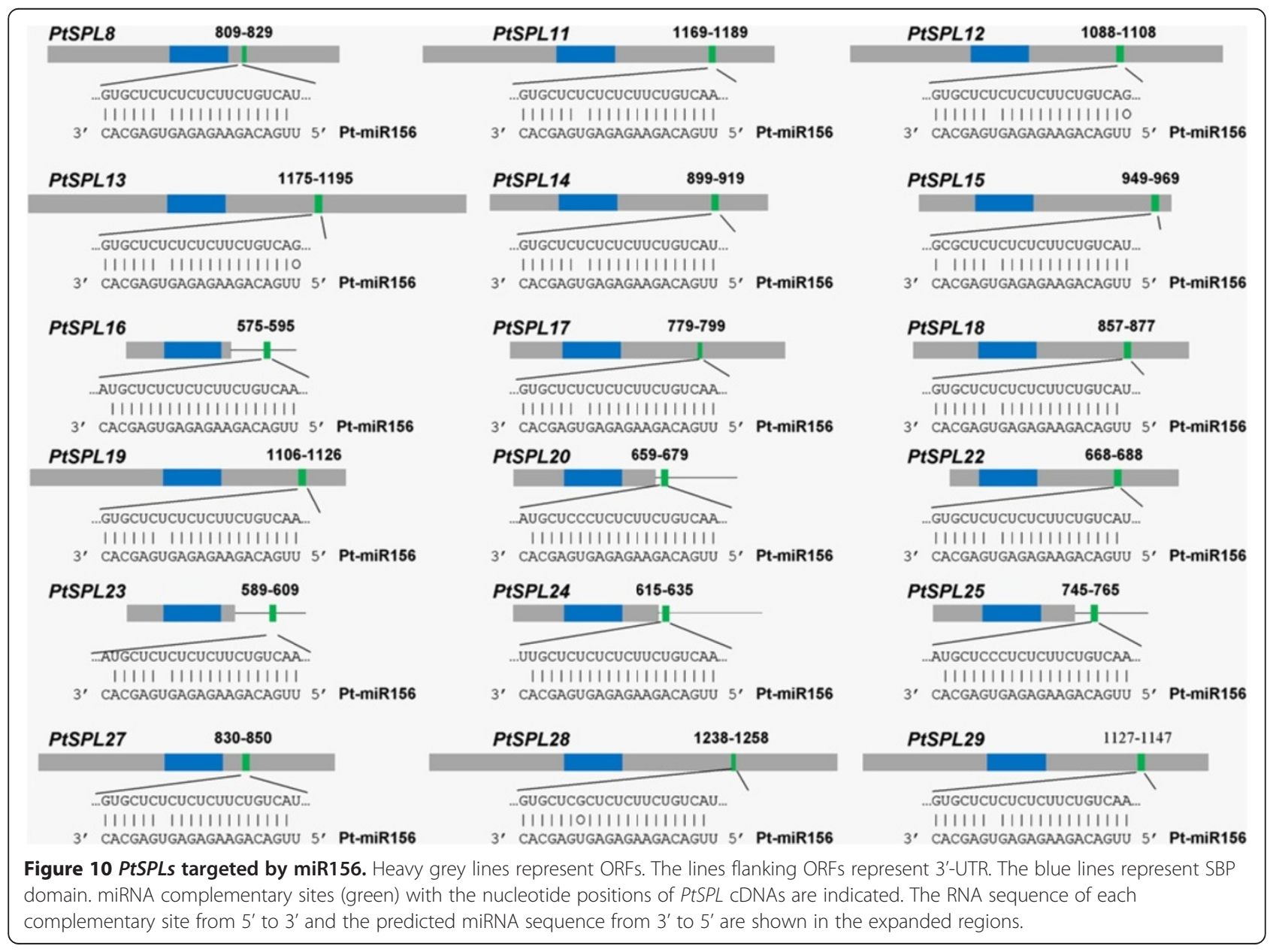




\section{Phylogenetic construction and motif analysis}

Phylogenetic trees were constructed using the neighborjoining (NJ) method in MEGA5.1. Branching reliability was assessed by the bootstrap re-sampling method using 1,000 bootstrap replicates. Only nodes supported by bootstrap values greater than $50 \%$ were analyzed. Conserved domains of PtSPLs were identified using Pfam (http://pfam.sanger. ac.uk) and by BLAST analysis of protein sequences against the Conserved Domain Database (CDD, http://www.ncbi. nlm.nih.gov/Structure/cdd/wrpsb.cgi) with the expected evalue threshold of 1.0 and the maximum size of hits to be 500 amino acids [70]. The 78 amino acids of SBP domain were aligned using clustalW. Sequence logos were generated using the weblogo platform (http://weblogo.berkeley. $\mathrm{edu} /$ ). Potential protein motifs were predicted using the MEME package (http://meme.sdsc.edu/meme/) with the following parameters applied. It includes the distribution of motifs: zero and one per sequence, maximum number of motifs to find: 25 , minimum width of motif: 8 , and maximum width of motif: 150 . An e-value cut off of $1 \mathrm{e}^{-10}$ was applied to the recognition.

\section{Quantitative real-time reverse transcription-PCR (qRT-PCR)}

$P$. trichocarpa plants were grown in an artificial climate chamber for about one year. Young leaves (2nd-3rd from the top), mature leaves (12th from the top), young stems (1st-3rd from the top), young roots, tissues of developing secondary xylem and phloem from the 4th-6th and 12th-25th internodes from the top of P. trichocarpa plants were collected. Three biological repeats were carried out. Total RNA was extracted using the plant total RNA extraction kit (Aidlab, China). Genomic DNA contamination was eliminated by pre-treating total RNA with RNase-free DNase (Promega, USA). RNA integrity was analyzed on a $1.2 \%$ agarose gel and its quantity was determined using a NanoDrop 2000C Spectrophotometer (Thermo Scientific, USA). Total RNA was reversetranscribed by Superscript III Reverse Transcriptase (Invitrogen, USA). qRT-PCRs were carried out in triplicate for each tissue sample using gene-specific primers (Additional file 6) as described previously [71]. The program used for qRT-PCR is as follows: predenaturation at $95^{\circ} \mathrm{C}$ for $30 \mathrm{~s}, 40$ cycles of amplification at $95^{\circ} \mathrm{C}$ for $5 \mathrm{~s}$, $60^{\circ} \mathrm{C}$ for $18 \mathrm{~s}$ and $72^{\circ} \mathrm{C}$ for $15 \mathrm{~s}$. The length of amplicons was between $80 \mathrm{bp}$ and $250 \mathrm{bp}$. Actin was used as a reference gene as described previously [72]. Dissociation curve was used to assess amplification specificity. Relative abundance of transcripts was analyzed using the comparative $\mathrm{Ct}$ method [73]. The arithmetic formula, $2-\Delta \Delta \mathrm{Cq}$, was used to achieve results for relative quantification. $\mathrm{Cq}$ represents the threshold cycle. Standardization of gene expression data from three biological replicates was performed as described [74]. For statistical analysis, ANOVA (analysis of variance) was calculated using SPSS (Version 19.0, IBM, USA). P < 0.05 was considered statistically significant.

\section{Microarray data analysis}

Microarray data of PtSPLs was obtained by the ePlanttissue expression tool at PopGenIE (http://www.popgenie. org/). The data was gene-wise normalized and then analyzed using the average linkage clustering technique in Cluster 3.0 [75].

\section{Prediction of PtSPLs targeted by miR156}

The sequences of $P$. trichocarpa miR156a-miR156j were obtained from miRBase [36] (http://www.mirbase.org/). PtSPLs targeted by miR156 were predicted by searching the coding regions and 3' UTRs of all PtSPLs for complementary sequences of $P$. trichocarpa miR156a-miR156j on the psRNATarget server using default parameters [45] (http:// plantgrn.noble.org/psRNATarget/?function=3).

\section{Availability of supporting data}

The data sets supporting the results of this article are included within the article and its additional files.

\section{Additional files}

Additional file 1: Sequence features of PtSPLs in P. trichocarpa. Protein length, intron number, $\mathrm{pl}$ and molecular weight of SPLs in $P$. trichocarpa are shown.

Additional file 2: Sequence features of AtSPLs in A. thaliana. Gene IDs, protein length, intron number, p/ and molecular weight of SPLs in A. thaliana are shown.

Additional file 3: Neighbor-joining (NJ) phylogenetic tree constructed for 77 SPLs from P. trichocarpa, Arabidopsis, rice and S. miltiorrhiza. The groups of homologous genes identified and bootstrap values are shown. The reliability of branching was assessed by the bootstrap re-sampling method using 1,000 bootstrap replicates. Bootstrap values are shown below nodes.

Additional file 4: Intron distribution on SBP domains of Populus and Arabidopsis. Intron distribution on SBP domains of Populus and Arabidopsis are shown.

Additional file 5: Alignment of the ANK/ANK-2 domain. The ANK ANK-2 domain is indicated by solid lines.

Additional file 6: Primers used for qRT-PCR analysis of PtSPL genes. Complete set of primers used for qRT-PCR.

\section{Abbreviations}

CURE: Cu-response element; Mw: The molecular weight; NJ: Neighbor-joining; NLS: Nuclear location signal; pl: Isoelectric point; qRT-PCR: Quantitative realtime reverse transcription-PCR; SBP: SQUAMOSA PROMOTER BINDING PROTEIN; Zn1: Zinc finger 1; Zn2: Zinc finger 1.

\section{Competing interests}

The authors declare that they have no competing interests.

\section{Authors' contributions}

$\mathrm{CL}$ contributed to bioinformatics and GRT-PCR analyses and participated in writing the manuscript. SL designed the experiment, performed bioinformatics analysis and wrote the manuscript. Both authors have read and approved the version of manuscript. 


\section{Acknowledgements}

This work was supported by grants from the National Key Basic Research Program of China (973 program) (2012CB114502 to S.L) and the Program for Xiehe Scholars in Chinese Academy of Medical Sciences \& Peking Union Medical College (to SL).

Received: 30 March 2014 Accepted: 6 May 2014

Published: 15 May 2014

\section{References}

1. Yamasaki K, Kigawa T, Inoue M, Tateno M, Yamasaki T, Yabuki T, Aoki M, Seki E, Matsuda T, Nunokawa E, Ishizuka Y, Terada T, Shirouzu M, Osanai T, Tanaka A, Seki M, Shinozaki K, Yokoyama S: A novel zinc-binding motif revealed by solution structures of DNA-binding domains of Arabidopsis SBP-family transcription factors. J Mol Biol 2004, 337:49-63.

2. Birkenbihl RP, Jach $G$, Saedler $H$, Huijser P: Functional dissection of the plant-specific SBP-domain: overlap of the DNA-binding and nuclear localization domains. J Mol Biol 2005, 352:585-596.

3. Klein J, Saedler H, Huijser P: A new family of DNA binding proteins includesputative transcriptional regulators of the Antirrhinum majus floral meristem identity gene SQUAMOSA. Mol Gen Genet 1996, 250:7-16.

4. Cardon G, Hohmann S, Klein J, Nettesheim K, Saedler H, Huijser P: Molecular characterisation of the Arabidopsis SBP-box genes. Gene 1999 237:91-104.

5. Arazi T, Talmor-Neiman M, Stav R, Riese M, Huijser P, Baulcombe DC: Cloning and characterization of micro-RNAs from moss. Plant J 2005, 43:837-848.

6. Hultquist JF, Dorweiler JE: Feminized tassels of maize mop1 and ts 1 mutants exhibit altered levels of miR156 and specific SBP-box genes. Planta 2008, 229:99-113.

7. Riese M, Zobell O, Saedler H, Huijser P: SBP-domain transcription factors as possible effectors of cryptochrome-mediated blue light signalling in the moss Physcomitrella patens. Planta 2008, 227:505-515.

8. Miura K, Ikeda M, Matsubara A, Song X, Ito M, Asano K, Matsuoka M, Kitano $H$, Ashikari M: OsSPL14 promotes panicle branching and higher grain productivity in rice. Nat Genet 2010, 42:545-549.

9. Salinas M, Xing S, Höhmann S, Berndtgen R, Huijser P: Genomic organization phylogenetic comparison and differential expression of the SBP-box family of transcription factors in tomato. Planta 2012, 235:1171-1184.

10. Cardon GH, Hohmann S, Nettesheim K, Saedler H, Huijser P: Functional analysis of the Arabidopsis thaliana SBP-box gene SPL3: a novel gene involved in the floral transition. Plant J 1997, 12:367-377.

11. Rhoades MW, Reinhart BJ, Lim LP, Burge CB, Bartel B, Bartel DP: Prediction of plant microRNA targets. Cell 2002, 110:513-520.

12. Kasschau KD, Xie Z, Allen E, Llave C, Chapman EJ, Krizan KA, Car-rington JC: P1/HC-Pro a viral suppressor of RNA silencing interferes with Arabidopsis development and miRNA function. Dev Cell 2003, 4:205-217.

13. Chen J, Li WX, Xie D, Peng JR, Ding SW: Viral virulence protein suppresses RNA silencing-mediated defense but upregulates the role of microrna in host gene expression. Plant Cell 2004, 16:1302-1313.

14. Schwab R, Palatnik JF, Riester M, Schommer C, Schmid M, Weigel D: Specific effects of microRNAs on the plant transcriptome. Dev Cell 2005, 8:517-527.

15. Wu G, Poethig RS: Temporal regulation of shoot development in Arabidopsis thaliana by miR156 and its target SPL3. Development 2006, 133:3539-3547.

16. Wang JW, Czech B, Weigel D: miR156-regulated SPL transcrip-tion factors define an endogenous flowering pathway in Arabidopsis thaliana. Cell 2009, 138:738-749.

17. Yu N, Cai WJ, Wang S, Shan CM, Wang LJ, Chen XY: Temporal control of trichome distribution by microRNA156-targeted SPL genes in Arabidopsis thaliana. Plant Cell 2010, 22:2322-2335.

18. Gandikota M, Birkenbihl RP, Höhmann S, Cardon GH, Saedler H, Huijser P. The miRNA156/157 recognition element in the $3^{\prime} U T R$ of the Arabidopsis SBP box gene SPL3 prevents early flowering by translational inhibition in seedlings. Plant J 2007, 49:683-693.

19. Shikata M, Koyama T, Mitsuda N, Ohme-Takagi M: Arabidopsis SBP-box genes SPL10, SPL11 and SPL2 control morphological change in association with shoot maturation in the reproductive phase. Plant Cell Physiol 2009, 50:2133-2145.
20. Vazquez F, Gasciolli V, Crété $P$, Vaucheret $H$ : The nuclear dsRNA binding protein HYL1 is required for microRNA accumulation and plant development but not posttranscriptional transgene silencing. Curr Biol 2004, 14:346-351.

21. Schwarz S, Grande AV, Bujdoso N, Saedler H, Huijser P: The microRNA regulated SBP-box genes SPL9 and SPL15 control shoot maturation in Arabidopsis. Plant Mol Biol 2008, 67:183-195.

22. Yamasaki H, Hayashi M, Fukazawa M, Kobayashi Y, Shikanai T: SQUAMOSA promoter binding protein-like7 is a central regulator for copper homeostasis in Arabidopsis. Plant Cell 2009, 21:347-361.

23. Unte US, Sorensen AM, Pesaresi P, Gandikota M, Leister D, Saedler H, Huijser P: SPL8 an SBP-Box gene that affects pollen sac development in Arabidopsis. Plant Cell 2003, 15:1009-1019.

24. Xing S, Salinas M, Höhmann S, Berndtgen R, Huijser P: miR156-targeted and nontargeted SBP-box transcription factors act in concert to secure male fertility in Arabidopsis. Plant Cell 2010, 22:3935-3950.

25. Zhang Y, Schwarz S, Saedler H, Huijser P: SPL8 a local regulator in a subset of gibberellins-mediated developmental processes in Arabidopsis. Plant Mol Biol 2007, 63:429-439.

26. Stone JM, Liang X, NekI ER, Stiers JJ: Arabidopsis AtSPL14 a plant-specific SBP-domain transcription factor participates in plant development and sensitivity to fumonisin B1. Plant J 2005, 41:744-754

27. Xie $K$, Wu $C$, Xiong L: Genomic organization differential expression and interaction of SQUAMOSA promoter-binding-like transcription factors and micro-RNA156 in rice. Plant Physiol 2006, 142:280-293.

28. Jiao Y, Wang Y, Xue D, Wang J, Yan M, Liu G, Dong G, Zeng D, Liu Z, Zhu X, Qian Q, Li J: Regulation of OsSPL14 by OsmiR156 definesideal plant architecture in rice. Nat Genet 2010, 42:541-544.

29. Wang S, Wu K, Yuan Q, Liu X, Liu Z, Lin X, Zeng R, Zhu H, Dong G, Qian Q, Zhang G, Fu X: Control of grain size shape and quality by OSSPL16 in rice. Nat Genet 2012, 44:950-955.

30. Becraft PW, Bongard-Pierce DK, Sylvester AW, Poethig RS, Freeling M: The liguleless-1 gene acts tissue specifically in maize leaf development. Dev Biol 1990, 141:220-232.

31. Moreno MA, Harper LC, Krueger RW, Dellaporta SL, Freeling M: liguleless 1 encodes a nuclear-localized protein required for induction of ligules and auricles during maize leaf organogenesis. Genes Dev 1997, 11:616-628.

32. Tuskan GA, DiFazio S, Jansson S, Bohlmann J, Grigoriev I, Hellsten U, Putnam N, Ralph S, Rombauts S, Salamov A, Schein J, Sterck L, Aerts A, Bhalerao RR, Bhalerao RP, Blaudez D, Boerjan W, Brun A, Brunner A, Busov V, Campbell M, Carlson J, Chalot M, Chapman J, Chen GL, Cooper D, Coutinho PM, Couturier J, Covert S, Cronk Q, et al: The genome of black cottonwood Populus trichocarpa (Torr. \& Gray). Science 2006, 313:1596-1604.

33. Lu S, Sun YH, Shi R, Clark C, Li L, Chang VL: Novel and mechanical stressresponsive microRNAs in Populus trichocarpa that are absent from Arabidopsis. Plant Cell 2005, 17:2186-2203.

34. Tuskan GA, DiFazio SP, Teichmann T: Poplar genomics is getting popular: the impact of the poplar genome project on tree research. Plant Biol 2004, 6:2-4.

35. Lu S, Sun YH, Chiang VL: Stress-responsive microRNAs in Populus. Plant J 2008, 55:131-151.

36. Kozomara A, Griffiths-Jones S: miRBase: integrating microRNA annotation and deep-sequencing data. Nucleic Acids Res 2011, 39:D152-D157.

37. Puzey JR, Karger A, Axtell M, Kramer EM: Deep annotation of Populus trichocarpa microRNAs from diverse tissue sets. PLoS One 2012, 7:e33034.

38. Lu S, Li Q, Wei H, Chang MJ, Tunlaya-Anukit S, Kim H, Liu J, Song J, Sun YH, Yuan L, Yeh TF, Peszlen I, Ralph J, Sederoff RR, Chiang VL: Ptr-miR397a is a negative regulator of laccase genes affecting lignin content in Populus trichocarpa. Proc Natl Acad Sci USA 2013, 110:10848-10853.

39. Shuai $P$, Liang D, Zhang Z, Yin W, Xia X: Identification of droughtresponsive and novel Populus trichocarpa microRNAs by highthroughput sequencing and their targets using degradome analysis. BMC Genomics 2013, 14:233.

40. Lu S, Yang C, Chiang VL: Conservation and diversity of microRNAassociated copper-regulatory networks in Populus trichocarpa. J Integr Plant Biol 2011, 53:879-891.

41. Zhang L, Wu B, Zhao D, Li C, Shao F, Lu S: Genome-wide analysis and molecular dissection of the SPL gene family in Salvia miltiorrhiza. J Integr Plant Biol 2013, 56:38-50.

42. Mosavi LK, Minor DL Jr, Peng ZY: Consensus-derived structural determinants of the ankyrin repeat motif. Proc Natl Aca Sci USA 2002, 99:16029-16034. 
43. Guo AY, Zhu QH, Gu X, Ge X, Yang J, Luo J: Genome-wide identification and evolutionary analysis of the plant specific SBP-box transcription factor family. Gene 2008, 418:1-8.

44. Sjödin A, Street NR, Sandberg G, Gustafsson P, Jansson S: The Populus Genome Integrative Explorer (PopGenIE): a new resource for exploring the Populus genome. New phytol 2009, 182:1013-1025.

45. Dai X, Zhao PX: psRNATarget: a Plant Small RNA Target Analysis Server. Nucleic Acids Res 2011, 39:W155-W159.

46. Huijser P, Klein J, Lonnig WE, Meijer H, Saedler H, Sommer H: Bracteomania an inflorescence anomaly is caused by the loss of function of the MADSbox gene SQUAMOSA in Antirrhinum majus. EMBO J 1992, 11:1239-1249.

47. Saedler H, Becker A, Winter KU, Kirchner C, Theissen G: MADS-box genes are involved infloral development and evolution. Acta Biochim Pol 2001, 48:351-358.

48. Fornara F, Parenicova L, Falasca G, Pelucchi N, Masiero S, Ciannamea S, Lopez-Dee Z, Altamura MM, Colombo L, Kater MM: Functional characterization of OsMADS18 a member of the AP1/SQUA subfamily of MADS box genes. Plant Physiol 2004, 135:2207-2219.

49. Robles P, Pelaz S: Flower and fruit development in Arabidopsis thaliana. Int J Dev Biol 2005, 49:633-643.

50. Shao CX, Takeda Y, Hatano S, Matsuoka M, Hirano HY: Rice genes encoding the SBP domain protein which is a new type of transcription factor controlling plant development. Rice Genet News/ 1999, 16:114.

51. Lännenpää M, Jänönen I, Hölttä-Vuori M, Gardemeister M, Porali I, Sopanen $\mathrm{T}$ : A new SBP-box gene BpSPL1 in silver birch (Betula pendula). Physiol Plant 2004, 120:491-500

52. Kent WJ, Baertsch R, Hinrichs A, Miller W, Haussler D: Evolution's cauldron: duplication deletion and rearrangement in the mouse and human genomes. Proc Natl Acad Sci USA 2003, 100:11484-11489.

53. Cannon SB, Mitra A, Baumgarten A, Young ND, May G: The roles of segmental and tandem gene duplication in the evolution of large gene families in Arabidopsis thaliana. BMC Plant Biol 2004, 4:10.

54. Du H, Yang SS, Liang Z, Feng BR, Liu L, Huang YB, Tang YX: Genome-wide analysis of the MYB transcription factor superfamily in soybean. BMC Plant Biol 2012, 12:106.

55. Zahn LM, Kong H, Leebens-Mack JH, Kim S, Soltis PS, Landherr LL, Soltis DE, Depamphilis CW, Ma H: The evolution of the SEPALLATA subfamily of MADS-box genes: a preangiosperm origin with multiple duplications throughout angiosperm history. Genetics 2005, 169:2209-2223.

56. Shigyo $M$, Hasebe $M$, Ito $M$ : Molecular evolution of the AP2 subfamily. Gene 2006, 366:256-265.

57. Blanc $\mathrm{G}$, Wolfe $\mathrm{KH}$ : Widespread paleopolyploidy in model plant species inferred from age distributions of duplicate genes. Plant Cell 2004, 16:1667-1678.

58. Bowers JE, Chapman BA, Rong J, Paterson AH: Unravelling angiosperm genome evolution by phylogenetic analysis of chromosomal duplication events. Nature 2003, 422:433-438.

59. Paterson $\mathrm{AH}$, Bowers JE, Chapman BA: Ancient polyploidization predating divergence of the cereals and its consequences for comparative genomics. Proc Natl Acad Sci USA 2004, 101:9903-9908.

60. Wang X, Shi X, Hao B, Ge S, Luo J: Duplication and DNA segmental loss in the rice genome: implications for diploidization. New Phytol 2005, 165:937-946.

61. Yang Z, Wang X, Gu S, Hu Z, Xu H, Xu C: Comparative study of SBP-box gene family in Arabidopsis and rice. Gene 2008, 407:1-11.

62. Bai J, Pennill LA, Ning J, Lee SW, Ramalingam J, Webb CA, Zhao B, Sun Q, Nelson JC, Leach JE, Hulbert SH: Diversity in nucleotide binding siteleucine-rich repeat genes in cereals. Genome Res 2002, 12:1871-1884.

63. Zhang S, Chen C, Li L, Meng L, Singh J, Jiang N, Deng XW, He ZH, Lemaux PG: Evolutionary expansion gene structure and expression of the rice wall- associated kinase gene family. Plant Physiol 2005, 139:1107-1124.

64. Jain M, Tyagi AK, Khurana JP: Genome-wide analysis evolutionary expansion and expression of early auxin-responsive SAUR gene family in rice (Oryza sativa). Genomics 2006, 88:360-371.

65. Wu G, Park MY, Conway SR, Wang JW, Weigel D, Poethig RS: The sequential action of miR156 and miR172 regulates developmental timing in Arabidopsis. Cell 2009, 138:750-759.

66. Shiu SH, Karlowski WM, Pan R, Tzeng YH, Mayer KF, Li WH: Comparative analysis of the receptor-like kinase family in Arabidopsis and rice. Plant Cell 2004, 16:1220-1234.
67. Librado P, Rozas J: DnaSP v5: a software for comprehensive analysis of DNA polymorphism data. Bioinformatics 2009, 25:1451-1452.

68. Bjellqvist B, Basse B, Olsen E, Celis JE: Reference points for comparisons of two-dimensional maps of proteins from different human cell types defined in a $\mathrm{pH}$ scale where isoelectric points correlate with polypeptide compositions. Electrophoresis 1994, 15:529-539.

69. Guo AY, Zhu QH, Chen X, Luo JC: GSDS: a gene structure display server. Yi Chuan 2007, 29:1023-1026.

70. Marchler-Bauer A, Lu S, Anderson JB, Chitsaz F, Derbyshire MK, DeWeese-Scott C, Fong JH, Geer LY, Geer RC, Gonzales NR, Gwadz M, Hurwitz DI, Jackson JD, Ke Z, Lanczycki CJ, Lu F, Marchler GH, Mullokandov M, Omelchenko MV, Robertson CL, Song JS, Thanki N, Yamashita RA, Zhang D, Zhang N, Zheng C, Bryant SH: CDD: A Conserved Domain Database for the functional annotation of proteins. Nucleic Acids Res 2011, 39:D225-D229.

71. Ma Y, Yuan L, Wu B, Li X, Chen S, Lu S: Genome-wide identification and characterization of novel genes involved in terpenoid biosynthesis in Salvia miltiorrhiza. J Exp Bot 2012, 63:2809-2823.

72. Su X, Fan B, Yuan L, Cui X, Lu S: Selection and validation of reference genes for quantitative RT-PCR analysis of gene expression in Populus trichocarpa. Chin Bull Bot 2013, 48:507-518.

73. Livak KJ, Schmittgen TD: Analysis of relative gene expression data using real-time quantitative PCR and the 2 (-Delta Delta $C(T)$ ) method. Methods 2001, 25:402-408.

74. Willems E, Leyns L, Vandesompele J: Standardization of real-time PCR gene expression data from independent biological replicates. Anal Biochem 2008, 379:127-129.

75. Eisen MB, Spellman PT, Brown PO, Botstein D: Cluster analysis and display of genome-wide expression patterns. Proc Natl Acad Sci USA 1998, 95:14863-14868.

doi:10.1186/1471-2229-14-131

Cite this article as: Li and Lu: Molecular characterization of the SPL gene family in Populus trichocarpa. BMC Plant Biology 2014 14:131.

\section{Submit your next manuscript to BioMed Central and take full advantage of:}

- Convenient online submission

- Thorough peer review

- No space constraints or color figure charges

- Immediate publication on acceptance

- Inclusion in PubMed, CAS, Scopus and Google Scholar

- Research which is freely available for redistribution

Submit your manuscript at www.biomedcentral.com/submit
C) BioMed Central 